FERNANDES, A.A.; MARTINEZ, H.E.P.; OLIVEIRA, L.R. Produtividade, qualidade dos frutos e estado nutricional de plantas de pepino, cultivadas em hidroponia, em função das fontes de nutrientes. Horticultura Brasileira, Brasília, v. 20, n. 4, p. 571-575, dezembro 2002.

\title{
Produtividade, qualidade dos frutos e estado nutricional de plantas de pepino, cultivadas em hidroponia, em função das fontes de nutrientes
}

\author{
Adriano A. Fernandes; Herminia Emilia P. Martinez; Lucimar R. de Oliveira \\ UFV, Depto. Fitotecnia, 36.571-000 Viçosa-MG; E-mail: aalves@alunos.ufv.br
}

\begin{abstract}
RESUMO
Avaliou-se a produtividade, qualidade dos frutos e estado nutricional de plantas de pepino cultivadas em soluções nutritivas preparadas com diferentes conjuntos de fontes de nutrientes. $\mathrm{O}$ experimento foi conduzido em estufa, avaliando-se dois conjuntos de fontes de nutrientes para compor as soluções nutritivas de crescimento vegetativo e dois conjuntos para compor as soluções nutritivas de frutificação. As combinações desses conjuntos deram origem a quatro tratamentos que foram dispostos no delineamento de blocos ao acaso com cinco repetições. As soluções nutritivas de crescimento vegetativo continham: $8 ; 2 ; 4 ; 2 ; 1$ e $1 \mathrm{mmol} \mathrm{L}^{-1}$ de $\mathrm{N}, \mathrm{P}, \mathrm{K}$, $\mathrm{Ca}, \mathrm{Mg}$ e S e $35 ; 19 ; 21 ; 4 ; 0,9$ e $0,7 \mathrm{mmol} \mathrm{L}^{-1}$ de Fe, Mn, B, Zn, Cu e Mo, respectivamente. As soluções nutritivas de frutificação continham: $12 ; 3 ; 8,6 ; 3 ; 1,5 ;$ e $1,5 \mathrm{mmol} \mathrm{L}^{-1}$ de N, P, K, Ca, Mg e S e 59 ; $28 ; 31 ; 4 ; 1,3$; e $0,7 \mathrm{mmol} \mathrm{L}^{-1}$ de Fe, Mn, B, Zn, Cu e Mo, respectivamente. As fontes de $\mathrm{P}$ empregadas foram fosfato monopotássico e ácido fosfórico. A variação na fonte de $\mathrm{P}$ implicou no uso de nitrato de sódio, cloreto de cálcio e cloreto de potássio, respectivamente, nos tratamentos 1 e 2; 3 e 4; e 2 e 4 . As mudas da cultivar Aodai, aos 21 dias após a semeadura (10 cm de comprimento), foram colocadas em vasos de $8,6 \mathrm{~L}$, no espaçamento de $0,40 \mathrm{~m}$ entre plantas e $0,70 \mathrm{~m}$ entre linhas. Avaliou-se o ciclo de cultivo, produção por planta, número, peso, matéria seca, comprimento, diâmetro, densidade e espessura da polpa dos frutos. Avaliou-se também a partição no fruto dos nutrientes $\mathrm{Ca}, \mathrm{Mg}$ e $\mathrm{K}$. Não foram encontradas diferenças significativas entre os tratamentos. As plantas produziram em média 13,2 frutos, com $18,10 \mathrm{~cm}$ de comprimento, $267,49 \mathrm{~g}$ de peso, 3,35\% de matéria seca, 4,93 cm de diâmetro, 942,6 $\mathrm{g} \mathrm{L}^{-1}$ de densidade e $2,3 \mathrm{~cm}$ de espessura da polpa. A produção média por planta foi de $3,46 \mathrm{~kg}$, com ciclo de 87 dias, correspondendo à produtividade média de $123 \mathrm{t}$ $\mathrm{ha}^{-1}$ no espaçamento adotado. Na análise foliar, foram obtidas para $\mathrm{N}$, $\mathrm{P}, \mathrm{K}, \mathrm{Ca}, \mathrm{Mg}$ e S as respectivas concentrações de 4,33; 0,77; 4,07; 6,$20 ; 1,42$; e 1,63 dag $\mathrm{kg}^{-1}$, e para Fe, $\mathrm{Mn}$ e $\mathrm{Cu}$ as respectivas concentrações de 288; 960 ; e $13 \mathrm{mg} \mathrm{kg}^{-1}$. O padrão de partição de Ca, Mg e K nos frutos não se manteve ao longo do tempo.
\end{abstract}

Palavras-chave: Cucumis sativus L, soluções nutritivas, nutrição mineral.

\begin{abstract}
Effect of nutrient sources on yield, fruit quality and nutritional status of cucumber plants, cultivated in hydroponics

Yield, fruit quality and nutritional status of cucumber plants cultivated in nutrient solutions, prepared with different nutrient sources, were evaluated. The experiment was carried out in greenhouse, being evaluated two groups of nutrient sources, to compose the nutritive solutions of vegetative growth and two groups to compose the nutritive solutions for fructification. The four treatments were disposed in the randomized blocks design with five replications. The nutritive solutions for vegetative growth were composed of $8 ; 2 ; 4 ; 2 ; 1$ and $1 \mathrm{mmol} \mathrm{L}^{-1}$ of $\mathrm{N}, \mathrm{P}, \mathrm{K}, \mathrm{Ca}, \mathrm{Mg}$ and $\mathrm{S}$ and $35 ; 19 ; 21 ; 4 ; 0.9$ and $0,7 \mathrm{mmol} \mathrm{L}^{-1}$ of Fe, Mn, B, Zn, Cu and Mo, respectively. The nutritive solutions for fructification were composed of $12 ; 3 ; 8.6 ; 3 ; 1.5$; and $1.5 \mathrm{mmol} \mathrm{L}^{-1}$ of $\mathrm{N}, \mathrm{P}, \mathrm{K}, \mathrm{Ca}, \mathrm{Mg}$ and $\mathrm{S}$, and $59 ; 28 ; 31 ; 4 ; 1.3$; and $0.7 \mathrm{mmol} \mathrm{L}^{-1}$ of Fe, $\mathrm{Mn}, \mathrm{B}, \mathrm{Zn}, \mathrm{Cu}$ and $\mathrm{Mo}$, respectively. The phosphorus sources were $\mathrm{KH}_{2} \mathrm{PO}_{4}$ and $\mathrm{H}_{3} \mathrm{PO}_{4}$. This variation implied in the use of $\mathrm{NaNO}_{3}, \mathrm{CaCl}_{2}$ and $\mathrm{KCl}$ in the treatments 1 and 2; 3 and 4; and 2 and 4. Aodai cultivar seedlings, 21 days after sowing date $(10 \mathrm{~cm}$ length), were grown in plastic recipient with $8.6 \mathrm{~L}$ of capacity, using a spacing of $0.40 \mathrm{x}$ $0.70 \mathrm{~m}$. Yield, fruit quality and plants nutritional status were evaluated, as well as the partition of $\mathrm{Ca}, \mathrm{Mg}$ and $\mathrm{K}$ in the fruit. There were no significant differences between treatments. The average yield was $3.46 \mathrm{~kg} \mathrm{plant}^{-1}$, crop cycle of 87 days corresponding to an average yield of $123 \mathrm{t} \mathrm{ha}^{-1}$ when considering the adopted spacing. Concentration of $\mathrm{N}, \mathrm{P}, \mathrm{K}, \mathrm{Ca}, \mathrm{Mg}$ and $\mathrm{S}$ in the leaves were 4.33; $0.77 ; 4.07 ; 6.20 ; 1.42$; and $1.63 \mathrm{dag} \mathrm{kg}^{-1}$, respectively. Concentration of $\mathrm{Fe}, \mathrm{Mn}$ and $\mathrm{Cu}$ in the leaves were 288; 960; and $13 \mathrm{mg} \mathrm{kg}^{-1}$, respectively. The partition pattern of $\mathrm{Ca}, \mathrm{Mg}$ and $\mathrm{K}$ in the fruits was not constant during the evaluation.
\end{abstract}

Keywords: Cucumis sativus L, nutritive solutions, mineral nutrition.

\section{(Recebido para publicação em 13 de dezembro de 2000 e aceito para publicação em 20 de maio de 2002 )}

A produção de plantas em sistema hidropônico tem como ponto chave a adequação da solução nutritiva à cultura de interesse. Várias fórmulas são propostas na literatura especializada, porém tem-se necessidade de associar a concentração da solução e a razão entre os nutrientes com crescimento e desenvolvimento da planta.

Em cultivos comerciais é comum o uso de apenas uma formulação de solu- ção nutritiva para diversos tipos de hortaliças de folhas. Quando isso ocorre com espécies que possuem relação de extração diferente, há possibilidade de desequilíbrio nutricional com acúmulo e/ou falta de nutrientes ao longo do ciclo da cultura, podendo comprometer a produção (Furlani et al. 1999b). Isso torna-se ainda mais significativo quando o trabalho é realizado com hortaliças de frutos, pois além da fase vegetativa acrescentam-se as fases de florescimento e de frutificação.

Para a produção de frutos na maioria das culturas, a relação entre $\mathrm{K}$ e $\mathrm{N}$ e, $\mathrm{K}$ e $\mathrm{P}$ deve ser diferente da utilizada para o desenvolvimento vegetativo (Furlani et al. 1999b; Adams, 1999). Segundo Furlani et al. (1999b), deve-se reduzir a relação N:K e aumentar P:K. Entretanto, de acordo com Adams (1994), as proporções de $\mathrm{N}$ e $\mathrm{K}$ absorvidas pela cultu- 
ra do pepino não se alteram com o início da frutificação, sendo de 1:1,3 durante todo o ciclo. Assim, estudos sobre a nutrição mineral de hortaliças cultivadas em hidroponia são essenciais para o estabelecimento das exigências nutricionais de cada espécie, tanto para as formulações das soluções nutritivas nos estádios vegetativo e reprodutivo quanto para a reposição periódica dos nutrientes durante o desenvolvimento da cultura.

Este trabalho teve como objetivo avaliar o efeito de soluções nutritivas preparadas com diferentes conjuntos de fontes de nutrientes na produtividade e qualidade de frutos e no estado nutricional das plantas de pepino.

\section{MATERIAL E MÉTODOS}

O experimento foi conduzido de 06/ 01 a 26/04/99. Foram avaliados dois conjuntos de fontes de nutrientes para compor as soluções nutritivas de crescimento vegetativo e dois conjuntos de fontes para compor as soluções nutritivas de frutificação (Tabela 1). As soluções nutritivas de crescimento vegetativo continham $8 ; 2 ; 4 ; 2 ; 1$; e 1 mmol L-1 de N, P, K, Ca, Mg e S, respectivamente, e $35 ; 19 ; 21 ; 4 ; 0,9$; e 0,7 mmol L-1 de Fe, Mn, B, Zn, Cu e Mo, respectivamente, e as soluções nutritivas de frutificação continham $12 ; 3 ; 8,6$; 3; 1,5; e 1,5 mmol L $\mathrm{m}^{-1}$ de N, P, K, Ca, $\mathrm{Mg}$ e $\mathrm{S}$ e $59 ; 28 ; 31 ; 4 ; 1,3$; e $0,7 \mathrm{mmol}$ $\mathrm{L}^{-1}$ de Fe, Mn, B, Zn, Cu e Mo, respectivamente. As combinações desses conjuntos deram origem a quatro tratamentos (Tratamento 1: Conjunto A $+\mathrm{C}$; Tratamento 2: Conjunto A $+\mathrm{D}$; Tratamento 3: Conjunto B + C e Tratamento 4: Conjunto $\mathrm{B}+\mathrm{D}$ ), cujos conjuntos estão definidos na Tabela 1. Os tratamentos foram dispostos no delineamento de blocos ao acaso com cinco repetições. As fontes de $\mathrm{P}$ empregadas foram fosfato monopotássico e ácido fosfórico. A variação na fonte de $\mathrm{P}$ implicou no uso de nitrato de sódio, cloreto de cálcio e cloreto de potássio, respectivamente, nos tratamentos 1 e $2 ; 3$ e 4 ; e 2 e 4 . A concentração de $\mathrm{Na}$ na fase de crescimento vegetativo, nos tratamentos $1 \mathrm{e}$ 2, foi de $1,7 \mathrm{mmol} \mathrm{L}^{-1}\left(39,2 \mathrm{mg} \mathrm{L}^{-1}\right)$. As concentrações de $\mathrm{Cl}$ foram de 0,3
mmol.L $\mathrm{L}^{-1}\left(10,7 \mathrm{mg} \mathrm{\textrm {L } ^ { - 1 }}\right)$ na fase vegetativa nos tratamentos 3 e 4, e 3,4 mmol.L $\mathrm{L}^{-1}\left(115,2 \mathrm{mg} \mathrm{L}^{-1}\right)$ na fase de frutificação nos tratamentos 2 e 4 .

As sementes de pepino (cv. Aodai) foram semeadas em copos plásticos de $50 \mathrm{ml}$ contendo vermiculita. Do $1^{\circ}$ ao $10^{\circ}$ dia, foram irrigadas com água desionizada; do $10^{\circ}$ ao 14을 dia, com solução de Steiner a $1 / 2$ força iônica e do $15^{\circ}$ ao transplante, irrigadas com solução de Steiner a 1 força iônica (Steiner, 1984). Todas essas irrigações foram realizadas uma vez ao dia, após as 18 horas, em volume suficiente para saturar o substrato e iniciar a percolação. Aos 21 dias quando as plantas atingiram de 10 a $15 \mathrm{~cm}$ foram selecionadas e transplantadas para vasos plásticos de 8,6 litros, com espaçamento de $0,40 \mathrm{~m}$ entre plantas e de $0,70 \mathrm{~m}$ entre linhas, contendo as soluções nutritivas que foram calculadas com base nos trabalhos de Adams (1994). A partir dessa data foram observadas diariamente as temperaturas máximas e mínimas no interior da estufa, por termômetro colocado fora de abrigo à altura de 1,10 m da superfície do piso.

Os conjuntos de fontes de nutrientes A e B utilizados na fase de crescimento vegetativo apresentaram inicialmente $\mathrm{pH}$ 6,10 e 3,38, respectivamente, enquanto que os conjuntos de fontes de nutrientes $\mathrm{C}$ e $\mathrm{D}$ utilizados na fase de frutificação, apresentaram os valores 6,13 e 2,98, respectivamente. $\mathrm{O} \mathrm{pH}$ das soluções nutritivas em todos os tratamentos foi monitorado e ajustado à faixa de 5,5 a 6,5 utilizando-se $\mathrm{HCl}$ ou $\mathrm{NaOH}$ (Martinez, 1999). Para reposição dos nutrientes, admitiu-se até $30 \%$ de depleção, com base na redução da condutividade elétrica inicial $(1,47$; 1,$46 ; 2,04$ e $2,40 \mathrm{mS} \mathrm{cm}^{-1}$ para os conjuntos de fontes de nutrientes A; B; C e $\mathrm{D}$, respectivamente). Durante o experimento, a evapotranspiração de cada vaso foi monitorada e medida com uma proveta volumétrica. As reposições foram realizadas com água desionizada admitindo-se uma redução máxima em torno de $40 \%$ do volume do vaso.

Aos 14 dias do transplantio (DAT), foi realizado o desbaste, retirando-se todas as brotações axilares até a sexta folha definitiva. A partir de 22 DAT foi realizada uma poda complementar deixando-se três folhas e dois frutos nos ramos secundários além do corte do meristema apical $1 \mathrm{~cm}$ acima da sexta folha definitiva. Durante a condução do experimento foram necessárias podas periódicas para manutenção do número de frutos e folhas preconizados. Aos 32 DAT foram trocadas as soluções de crescimento vegetativo pelas soluções de frutificação.

Para sustentar as plantas foram utilizadas tampas de isopor revestidas com papel alumínio. As tampas tinham um orifício central para o encaixe de espuma que serviu de suporte e proteção para a planta. A oxigenação das soluções nutritivas foi feita por compressor de ar.

Devido à ocorrência de oídio aplicou-se fungicida (Saprol $1 \mathrm{ml} \mathrm{L}^{-1}$ ) nos dias 03/03 e 21/03/99.

A colheita foi realizada dos 42 aos 87 DAT. No início e meio da frutificação foram amostrados dois frutos por planta. No primeiro fruto de cada amostragem foram avaliados matéria fresca e seca, comprimento, diâmetro, espessura da polpa, densidade e teor de nutrientes ( $\mathrm{Ca}, \mathrm{Mg}$ e K). Para a análise do teor de nutrientes, o fruto foi dividido transversalmente em três partes: proximal, mediana e distal, em relação ao pedúnculo. Nessa característica, os dados obtidos foram analisados em parcelas subdivididas, sendo as parcelas compostas pelas soluções nutritivas e as subparcelas pelas porções dos frutos. No segundo fruto foram avaliados peso da matéria fresca, comprimento, diâmetro e densidade. Nos demais frutos foi avaliado o peso da matéria fresca para obtenção da produção. Para a diagnose foliar, foi realizada uma amostragem da quinta folha desenvolvida a partir do ápice do caule principal aos 42 DAT, conforme o preconizado por Jones Junior et al. (1991).

Para a análise química, o material foi lavado em água desionizada e seco em estufa com circulação forçada de ar a $70^{\circ} \mathrm{C}$, até peso constante. Em seguida, foi pesado para a obtenção da matéria seca, moído em moinho tipo Wiley, equipado com peneira de 20 mesh. O $\mathrm{N}$-total foi determinado pelo método Kjeldahl descrito por Bremner (1965). Os demais elementos foram analisados 
após mineralização pela digestão nítrico-perclórica. O P foi dosado colorimetricamente pelo método de redução do fosfomolibdato pela vitamina C, conforme Braga \& Defelipo (1974); o K, por fotometria de emissão de chama; o $\mathrm{Ca}, \mathrm{Mg}, \mathrm{Fe}, \mathrm{Mn}, \mathrm{Zn}$ e $\mathrm{Cu}$, por espectrofotometria de absorção atômica; e o S, determinado por turbidimetria do sulfato (Blanchar et al., 1965).

Os dados foram submetidos à análise de variância e os efeitos dos tratamentos foram comparados pelo teste de Tukey a 5\% de probabilidade.

\section{RESULTADOS E DISCUSSÃO}

Durante o experimento as temperaturas médias semanais apresentaram mínima entre 10,0 e $13,7^{\circ} \mathrm{C}$ e máxima entre 19,8 e $32,0^{\circ} \mathrm{C}$. A variação na temperatura aparentemente não afetou nem o florescimento nem a produção de frutos, pois de acordo com o sistema de condução, houve necessidade da retirada do excesso de frutos. Observa-se que as temperaturas mínimas estão abaixo do considerado ideal por Papadopoulos (1994), o que certamente afetou a velocidade de crescimento das plantas e o ciclo da cultura. O consumo de água pelas plantas foi mais acentuado no período de frutificação atingindo o máximo de 13,2 litros por planta por semana. Os maiores consumos totais, obtidos através da soma das médias de consumo semanais, foram observados para os tratamentos $1(78,7 \mathrm{~L})$ e $3(78,1 \mathrm{~L})$ compostos pelo conjunto $\mathrm{C}$ de fontes de nutrientes. Esse consumo pode ser explicado em função dos valores da condutividade elétrica. Como o conjunto $\mathrm{C}$ possui menor $\mathrm{CE}\left(2,0 \mathrm{mS} \mathrm{cm} \mathrm{cm}^{-1}\right)$ que $\mathrm{D}\left(2,4 \mathrm{mS} \mathrm{cm}^{-1}\right)$ teria maior potencial hídrico que este último possibilitando maior absorção de água pelas plantas.

Para as características produção $(3,1$ a $3,7 \mathrm{~kg} /$ planta), número de frutos $(11,4$ a 14,0 frutos/planta), peso/fruto $(261,7$ a $270,9 \mathrm{~g})$, matéria seca/fruto $(3,1$ a 3,8 g), comprimento dos frutos $(17,5$ a 18,4 $\mathrm{cm})$, diâmetro dos frutos (4,9 a 5,0 cm), densidade dos frutos $(932,8$ a $955,9 \mathrm{~g}$ $\left.\mathrm{L}^{-1}\right)$ e espessura da polpa dos frutos $(2,2$ a 2,3 cm) não foram encontradas diferenças estatísticas significativas entre os tratamentos, o que indica que concen-

Tabela 1. Conjuntos de fontes de nutrientes componentes das soluções nutritivas utilizadas nas fases de crescimento vegetativo e de frutificação do pepino. Viçosa, UFV, 1999.

\begin{tabular}{lclc}
\hline \multicolumn{3}{c}{ Fase de crescimento vegetativo } \\
\hline \multicolumn{1}{c}{ Conjunto $\mathbf{A}$} & \multicolumn{1}{c}{ g 1000L-1 } & \multicolumn{1}{c}{ Conjunto B } & g 1000L-1 \\
\hline $\mathrm{KH}_{2} \mathrm{PO}_{4}$ & 272,00 & $\mathrm{H}_{3} \mathrm{PO}_{4}$ & 115,84 \\
$\mathrm{MgSO}_{4}$ & 246,40 & $\mathrm{MgSO}_{4}$ & 246,40 \\
$\mathrm{KNO}_{3}$ & 213,60 & $\mathrm{KNO}_{3}$ & 427,30 \\
$\mathrm{Ca}\left(\mathrm{NO}_{3}\right)_{2}$ & 421,00 & $\left.\mathrm{Ca}_{2} \mathrm{NO}_{3}\right)_{2}$ & 389,30 \\
$\mathrm{NaNO}_{3}$ & 144,90 & $\mathrm{CaCl}_{2}$ & 33,10 \\
$\mathrm{FeCl}_{3}$ & 9,60 & $\mathrm{FeCl}_{3}$ & 9,60 \\
$\mathrm{Na}_{2}-\mathrm{EDTA}$ & 13,24 & $\mathrm{Na}_{2}-\mathrm{EDTA}$ & 13,24 \\
$\mathrm{MnSO}_{4}$ & 3,70 & $\mathrm{MnSO}_{4}$ & 3,70 \\
$\mathrm{H}_{3} \mathrm{BO}_{3}$ & 1,30 & $\mathrm{H}_{3} \mathrm{BO}_{3}$ & 1,30 \\
$\mathrm{ZnSO}_{4}$ & 1,31 & $\mathrm{ZnSO}_{4}$ & 1,31 \\
$\mathrm{Na}_{2} \mathrm{MoO}_{4}$ & 0,18 & $\mathrm{Na}_{2} \mathrm{MoO}_{4}$ & 0,18 \\
$\mathrm{CuSO}_{4}$ & 0,13 & $\mathrm{CuSO}_{4}$ & 0,13 \\
\hline
\end{tabular}

Fase de Frutificação

\begin{tabular}{lclc}
\hline \multicolumn{1}{c}{ Conjunto C } & \multicolumn{1}{c}{ g 1000L-1 } & \multicolumn{1}{c}{ Conjunto D } & g 1000L-1 \\
\hline $\mathrm{KH}_{2} \mathrm{PO}_{4}$ & 408,70 & $\mathrm{H}_{3} \mathrm{PO}_{4}$ & 173,70 \\
$\mathrm{MgSO}_{4}$ & 369,60 & $\mathrm{MgSO}_{4}$ & 369,60 \\
$\mathrm{KNO}_{3}$ & 596,98 & $\mathrm{KNO}_{3}$ & 587,80 \\
$\mathrm{Ca}\left(\mathrm{NO}_{3}\right)_{2}$ & 631,58 & $\mathrm{Ca}\left(\mathrm{NO}_{3}\right)_{2}$ & 631,56 \\
& & $\mathrm{KCl}$ & 241,76 \\
$\mathrm{FeCl}_{3}$ & 15,92 & $\mathrm{FeCl}_{3}$ & 15,92 \\
$\mathrm{Na}_{2}-\mathrm{EDTA}$ & 20,00 & $\mathrm{Na}_{2}-\mathrm{EDTA}$ & 20,00 \\
$\mathrm{MnSO}_{4}$ & 5,48 & $\mathrm{MnSO}_{4}$ & 5,48 \\
$\mathrm{H}_{3} \mathrm{BO}_{3}$ & 1,91 & $\mathrm{H}_{3} \mathrm{BO}_{3}$ & 1,91 \\
$\mathrm{ZnSO}_{4}$ & 1,30 & $\mathrm{ZnSO}_{4}$ & 1,30 \\
$\mathrm{Na}_{2} \mathrm{MoO}_{4}$ & 0,18 & $\mathrm{Na}_{2} \mathrm{MoO}_{4}$ & 0,18 \\
$\mathrm{CuSO}_{4}$ & 0,18 & $\mathrm{CuSO}_{4}$ & 0,18 \\
\hline
\end{tabular}

trações de $39,2 \mathrm{mg} \mathrm{L}^{-1}\left(1,7 \mathrm{mmol} \mathrm{L}^{-1}\right)$ de Na na fase de crescimento vegetativo e $115,2 \mathrm{mg} \mathrm{L}^{-1}\left(3,4 \mathrm{mmol} \mathrm{L}^{-1}\right)$ de $\mathrm{Cl}$ na fase de frutificação não foram prejudiciais ao cultivo de pepino da cultivar Aodai. Nerson et al. (1997), também mostra que não há diferença entre a eficiência do $\mathrm{KH}_{2} \mathrm{PO}_{4}$ e a combinação de $\mathrm{H}_{3} \mathrm{PO}_{4}+\mathrm{KCl}$ para o cultivo do pepino.

Embora na literatura, para a cultura do pepino, não se tenha referência da necessidade de mudar a relação $\mathrm{N}: \mathrm{K}$ na fase de frutificação, houve bom desempenho com alteração da razão $\mathrm{N}: \mathrm{K}$ de $1: 1,4$ na fase vegetativa para $1: 2,0$ na fase reprodutiva. Tal relação foi empregada, visando avaliar a possibilidade de recomendar uma mesma solução nutritiva para cultivo de diferentes hortali- ças de frutos. Nesse caso, as soluções utilizadas para o crescimento e desenvolvimento do pepino, foram calculadas com base em recomendações para tomate (Adams, 1994).

A produção média por planta foi de $3,5 \mathrm{~kg}$, com um ciclo médio de 87 dias, o que corresponde à produtividade média de $123 \mathrm{tha}^{-1}$ ou $518 \mathrm{tha}^{-1}$ ano $^{-1}$, considerando o espaçamento adotado. Filgueira (1981) considera boa produção a campo para a cultivar Aodai, quando se produz de 10-15 frutos por planta, obtendo-se de 70-100 t ha-1. De acordo com os resultados, as plantas produziram em média 13,2 frutos, com $18,1 \mathrm{~cm}$ de comprimento, $267,5 \mathrm{~g}$ de peso, $3,4 \%$ de matéria seca, $4,9 \mathrm{~cm}$ de diâmetro, 942,6 $\mathrm{g} \mathrm{L}^{-1}$ de densidade e $2,3 \mathrm{~cm}$ de 
Tabela 2. Concentrações de $\mathrm{Ca}, \mathrm{Mg}$ e $\mathrm{K}$ nas porções proximal, mediana e distal de frutos de pepino, cultivados em hidroponia em função dos tratamentos nutricionais na primeira avaliação. Viçosa, UFV, 1999.

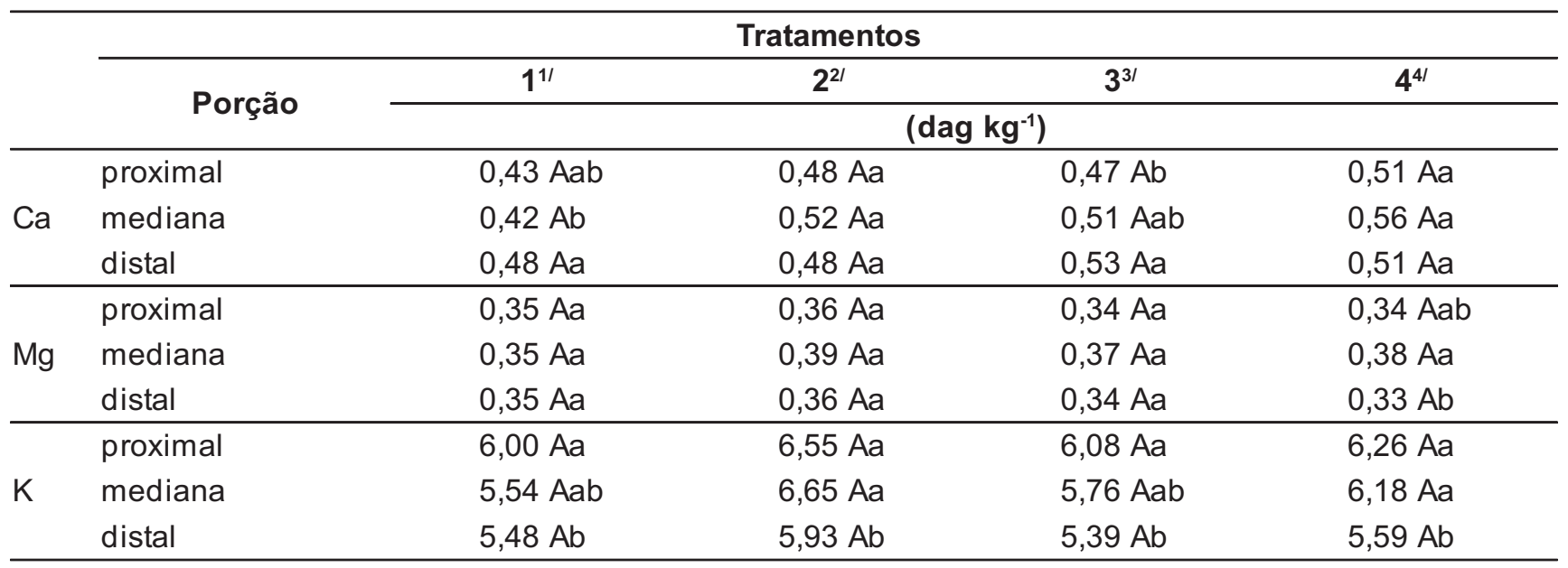

Médias seguidas pela mesma letra maiúscula nas linhas e minúscula nas colunas, não diferem entre si, a $5 \%$ de probabilidade, pelo teste de Tukey.

${ }^{1 /}$ Combinação das fontas de nutrientes dos conjuntos A e C da tabela $1 .{ }^{2 /}$ Combinação das fontas de nutrientes dos conjuntos A eD da tabela $1 .{ }^{3 /}$ Combinação das fontas de nutrientes dos conjuntos $\mathrm{B}$ e $\mathrm{C}$ da tabela $1 .{ }^{4 /}$ Combinação das fontas de nutrientes dos conjuntos $\mathrm{B}$ e $\mathrm{D}$ da tabela 1.

espessura da polpa. $\mathrm{O}$ comprimento e o peso dos frutos encontram-se abaixo dos valores citados por Filgueira (1981) e Gomes (1995), contudo houve um efeito compensatório pelo diâmetro e pelo número de frutos por planta, o que proporcionou uma boa produção. Para a maioria das características analisadas observou-se um baixo coeficiente de variação $(\mathrm{CV})$, o que demonstra a uniformidade obtida usando-se o cultivo hidropônico (2,2 a 27,8\%).

As concentrações médias de $\mathrm{N}, \mathrm{P}, \mathrm{K}$, $\mathrm{Fe}, \mathrm{Zn}$ e $\mathrm{Cu}$ foram de 4,3; 0,8; 4,1 dag $\mathrm{kg}^{-1}$ e 287,$5 ; 127,5$; e $13,0 \mathrm{mg} \mathrm{kg}^{-1}$, respectivamente, e estão de acordo com o indicado como adequado por Jones Junior et al. (1991). As concentrações médias de $\mathrm{Ca}, \mathrm{Mg}$, $\mathrm{S}$ e $\mathrm{Mn}$ foram 6,2; 1,$4 ; 1,6 \mathrm{dag} \mathrm{kg}^{-1}$ e $960 \mathrm{mg} \mathrm{kg}^{-1}$, respectivamente, estando acima da faixa considerada ideal por Jones Junior et al. (1991). Solís (1982) estabelece faixas de valores dos nutrientes para folhas sadias de pepino em dag $\mathrm{kg}^{-1}$ de 2,9 a 4,2 para N; 0,2 a 0,4 para $\mathrm{P} ; 1,6$ a 2,7 para $\mathrm{K} ; 0,6$ a 1,6 para $\mathrm{Ca} ; 0,9$ a 1,2 para $\mathrm{Mg}$; e 0,4 a 0,42 para S, e em $\mathrm{mg} \mathrm{kg}^{-1} \mathrm{de}$ 151 a 180 para Fe e 48,2 a 53,2 para Zn. Considerando-se essas faixas de valores, todas as concentrações de macro e micronutrientes encontradas são superiores. Essa variação ocorre porque o cultivo hidropônico proporciona maior absorção de nutrientes pelas plantas quando comparado aos cultivos convencionais, podendo-se estabelecer níveis críticos superiores para esses produtos.

As soluções nutritivas de crescimento vegetativo empregadas neste trabalho apresentam concentrações de $\mathrm{Ne} \mathrm{Ca}$ relativamente baixas quando comparadas às concentrações recomendadas por outros autores para a cultura do pepino (Martinez, 1997; Furlani et al., 1999a). As soluções de frutificação por sua vez, apresentam concentrações adequadas de macronutrientes, com exceção de $\mathrm{P}$ e K que são mais elevadas. Assim, as elevadas concentrações foliares de $\mathrm{Ca}, \mathrm{Mg}$ e $\mathrm{S}$ não podem ser justificadas com base na composição da solução nutritiva.

A concentração de Ca obtida para as folhas foi de $6,2 \mathrm{dag} \mathrm{kg}^{-1}$. Jones Junior et al. (1991) consideram ideal a faixa de 1,5 a $4,0{\text { dag } \mathrm{kg}^{-1}}^{\text {, }}$ porém em seu padrão de amostragem determina a colheita das folhas no estádio de frutos pequenos até a colheita, tendo assim um período longo e propício às variações. Ingestad (1973) relata que pode ocorrer acúmulo de $\mathrm{Ca}$ em folhas velhas de pepino, sendo que este mecanismo opera até mesmo com baixas taxas de absorção de $\mathrm{Ca}$.

Para os nutrientes avaliados, apenas as médias referentes às concentrações de $\mathrm{Zn}$ foram influenciadas pelos tratamentos, sendo que os tratamentos $1\left(117 \mathrm{mg} \mathrm{kg}^{-1}\right)$ que não possui $\mathrm{Cl}$, e $3\left(109 \mathrm{mg} \mathrm{kg}^{-1}\right)$ que apresenta $\mathrm{Cl}$ em menor quantidade, apresentaram plantas com menores teores de $\mathrm{Zn}$. Apenas o tratamento $2\left(155 \mathrm{mg} \mathrm{kg}^{-1}\right)$ apresentou diferença significativa em comparação aos tratamentos 1 e 3 . O tratamento 4 resultou em $129 \mathrm{mg} \mathrm{kg}^{-1}$.

As plantas apresentaram grande concentração de Mn, sendo a média de 960 $\mathrm{mg} \mathrm{kg}^{-1}$. Sintomas de toxidez de Mn em pepino aparecem quando as concentrações alcançam 500 e $800 \mathrm{mg} \mathrm{kg}^{-1} \mathrm{em}$ folhas jovens e velhas, respectivamente, e significante perda na produção pode ocorrer quando as concentrações atingirem 2.000 e $5.000 \mathrm{mg} \mathrm{kg}^{-1} \mathrm{em}$ folhas jovens e velhas, respectivamente (Papadopoulos, 1994). Horiguchi (1987) sugere mecanismos de tolerância do pepino ao excesso de Mn, através da oxidação do nutriente pelas raízes e pela excreção de Mn oxidado pelos tricomas das folhas.

Apesar dos valores elevados de $\mathrm{Mn}$ obtidos na matéria seca no presente estudo, as concentrações de micronutrientes empregadas na solução nutritiva situam-se na faixa preconizada para a cultura em outros trabalhos (Martinez, 1997; Furlani et al., 1999a).

Na primeira avaliação, entre 12 e 26 de março, houve uma maior tendência ao acúmulo de Ca na porção distal dos fru- 
Tabela 3. Concentrações de $\mathrm{Ca}, \mathrm{Mg}$ e $\mathrm{K}$ nas porções proximal, mediana e distal de frutos de pepino cultivados em hidroponia em função dos tratamentos nutricionais na segunda avaliação. Viçosa, UFV, 1999.

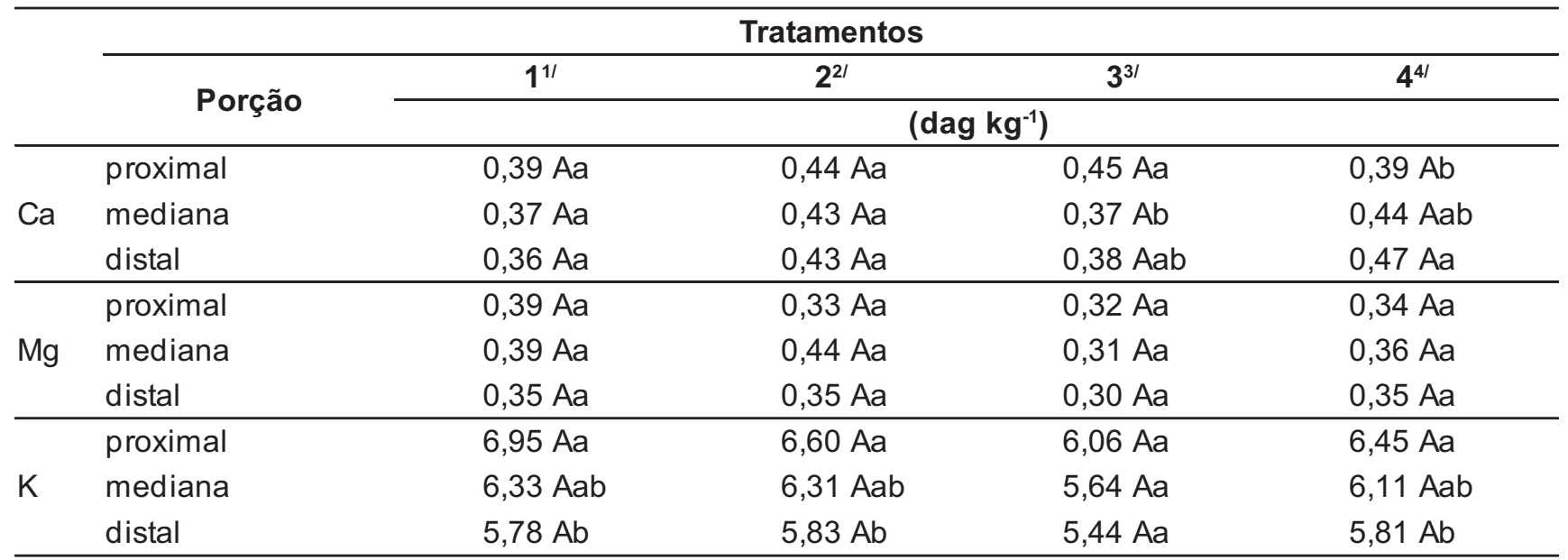

Médias seguidas pela mesma letra maiúscula nas linhas e minúscula nas colunas, não diferem entre si, a 5\% de probabilidade, pelo teste de Tukey.

${ }^{1 /}$ Combinação das fontas de nutrientes dos conjuntos $\mathrm{A}$ e $\mathrm{C}$ da tabela $1 .{ }^{2 /}$ Combinação das fontas de nutrientes dos conjuntos A eD da tabela $1 .{ }^{3 /}$ Combinação das fontas de nutrientes dos conjuntos B e C da tabela $1 .{ }^{4 /}$ Combinação das fontas de nutrientes dos conjuntos $\mathrm{B}$ e $\mathrm{D}$ da tabela 1.

tos pelos tratamentos 1 e 3 , maior acúmulo de $\mathrm{Mg}$ na porção mediana pelo tratamento 4 e maior acúmulo de $\mathrm{K}$ nas porções proximal e mediana dos frutos (Tabela 2). Pode-se observar que o fruto acumula quantidades razoáveis de Ca e apresenta, de certa forma, uma boa distribuição do elemento. Adams \& Ho (1995) obtiveram concentração de $\mathrm{Ca}$ em frutos de pepino, aproximadamente 6 vezes maior que a observada em frutos de tomate. Essa diferença, segundo os autores, é refletida pela maior densidade de estômatos nos frutos de pepino, sugerindo que a transpiração do fruto tem importante papel na concentração de Ca nos diferentes órgãos da planta.

O padrão de partição de $\mathrm{Ca}, \mathrm{Mg}$ e K nos frutos não se manteve ao longo do tempo. Na segunda avaliação, no período de 25 de março a 14 de abril, realizada no meio do período de frutificação, as concentrações de $\mathrm{Ca}$ nas diferentes porções foram influenciadas pelas soluções 3 e 4 . O tratamento 3 promoveu maior acúmulo de $\mathrm{Ca}$ na porção proximal e o tratamento 4 na porção distal. A concentração de $\mathrm{Mg}$ não variou com as diferentes soluções e tampouco nas partes do fruto na segunda avaliação. A concentração de K apresentou tendência de se reduzir da porção proximal para a distal (Tabela 3).

As concentrações dos nutrientes nas folhas não foram influenciadas pelos tratamentos. Os conjuntos de fontes de nutrientes foram apropriados ao cultivo do pepino, podendo-se selecionar o mais adequado em função do custo.

\section{AGRADECIMENTOS}

Á UFV pela oportunidade de realização do trabalho e a CAPES pela concessão da bolsa de pesquisa ao primeiro autor.

\section{LITERATURA CITADA}

ADAMS, P. Nutrition of greenhouse vegetables in NFT and hydroponic systems. Acta Horticulturae, n. 361, p. 245-257, 1994.

ADAMS, P.; HO, L.C. Differential effects of salinity and humidity on growth and Ca status of tomato and cucumber grown in hydroponic culture. In: GROWING MEDIA E PLANT NUTRITION. Acta Horticulturae, n. 401, p. 357363, 1995.

ADAMS, P. Plant nutrition demystified. Acta Horticulturae, n. 481, p. 341-344, 1999.

BLANCHAR, R.W.; REHM,G.; CALDWELL, A.C. Sulfur in plant material by digestion with nitric and perchloric acid. Proceedings-Soil Science Society of America, v. 29, n. 1, p.71-72, 1965.

BRAGA, J.M.; DEFELIPO, B.V. Determinação espectrofotométrica de fósforo em extratos de solos e plantas. Revista Ceres, Viçosa, v. 1, n. 113, p.73-85, 1974.

BREMNER, J.M. Total nitrogen In: BLACK, C.A. ed., Methods of soil analysis. Madison: American Society of Agronomy, 1965. part 2, p. 1149-1178. FILGUEIRA, F.A.R. Manual de olericultura: cultura e comercialização de hortaliças. 2. ed. São Paulo: Agronômica Ceres, 1981. 338 p.

FURLANI, P.R.; SILVEIRA, L.C.P.; BOLONHEZI, D.; FAQUIN, V. Cultivo hidropônico de plantas. Campinas: Instituto Agronômico, 1999a. 52 p. (Boletim Técnico, n.180).
FURLANI, P.R.; BOLONHEZI, D.; SILVEIRA, L.C.P.; FAQUIN, V. Nutrição mineral de hortaliças, preparo e manejo de soluções nutritivas. Informe Agropecuário, Belo Horizonte, v. 20, n. 200/ 201, p. 90-98, 1999b.

GOMES, S.M.S. Influência da idade, coloração externa e armazenamento de frutos na qualidade de sementes de pepino (Cucumis sativus L.). Viçosa: UFV, 1995. 80 p. (Tese mestrado).

HORIGUCHI, T. Mechanism of manganese toxicity and tolerance of plants. Soil Science Plant Nutrition, v. 33, n. 4, p. 595-606, 1987.

INGESTAD, T. Mineral nutrient requirents of cucumber seedlings. Plant Physiology, v. 52, n. 4, p. 332-338, 1973.

JONES JUNIOR, J.B.; WOLF, B.; MILLS, H.A. Plant analysis handbook, a practical sampling, preparation, analysis, and interpretation guide. Georgia, USA: Micro-Macro, 1991. 183 p.

MARTINEZ, H.E.P. Formulação de soluções nutritivas para cultivos hidropônicos comerciais. Jaboticabal: FUNEP, 1997. 31 p. (Boletim).

MARTINEZ, H.E.P. O uso do cultivo hidropônico de plantas em pesquisa. 2. ed. Viçosa: Imprensa Universitária, 1999. 47 p. (Cadernos Didáticos 1). NERSON, H.; EDELSTEIN, M.; BERDUGO, R.; ANKORION, Y. Monopotassium phosphate as a phosphorus and potassium source for greenhousewinter grown cucumber and muskmelon. Journal of Plant Nutrition, v. 20, n. 2/3, p. 335-344, 1997. PAPADOPOULOS, A.P. Growing greenhouse seedles cucumbers in soil and in soiless media. Canada: Agriculture and Agri-Food Canada, 1994. 126 p.

SOLÍS, F.A.M. Concentração e extração de nutrientes e distúrbios nutricionais na cultura de pepino (Cucumis sativus L.) variedade Aodai. Piracicaba: ESALQ, 1982. 139 p. (Tese doutorado).

STEINER, A.A. The universal nutrient solution. In: INTERNATIONAL CONGRESS ON SOILLESS CULTURE, 6., 1984, Lunteren. Proceedings...Lunteren: International Society of Soiless Culture, 1984. p. 633-649. 